\title{
UN CAS DE DISTOMATOSE SOUS-CUTANÉE AU MAROC
}

\author{
Par M. DEKESTER
}

L'existence de localisations erratiques des douves est signalée dans tous les traités de Pathologie exotique et de Parasitologie, mais les observations en restent exceptionnelles, aussi croyonsnous utile de relater l'observation suivante :

P..., 40 ans, gardien de prison, habitant Fès-Djedi, né en France, est arrivé à Fès, il y a 18 mois; il se présente à l'Hôpital Cocar'd le 17 décembre 1923 pour une petite lésion qui a commençé à apparaitre un mois et demi auparavant et dont l'extension l'inquiète. Ce malade est de complexion plutôt faible et donne l'impression d'un phtisique chronique. Pourtant il nous dit n'avoir jamais été malade. Une période de travail intense, il y a un an, a obligé le malade à séjourner un mois à l'hôpital pour prendre du repos; il n'a jamais craché de sang, n'a jamais eu d'ictère, de paludisme, ni de dysenterie. Foie et rate normaux. Il est marié et père de famille.

Le médecin-major Christiani, ayant examiné le malade, suppose une cause parasitaire et nous l'envoie. La lésion siège sur la partie inférieure de l'abdomen, à droite, parallèle à l'arcade crurale, mais à $5 \mathrm{~cm}$. at-dessus d'elle, commençant à 7-8 cm. environ en avant de l'épine illia. qque antérieure droite et se continuant à $5 \mathrm{~cm}$. en arrière d'elle. L'extrémité antéro-inférieure forme un petit nodule légèrement surélevé à aspect rougeâtre, un peu vineux, inflammatoire dont la partie centrale présente un point hémorragique assez analogue à une piqûre de puce ; une minuscule croutelle se trouve à l'extrémité inférieure de ce nodule, elle n'existe que depuis quelques jours et est due d'après le malade au grattage. Ce nodule est resté unique pendant une quinzaine de jours, étant lc siège de poussées intermittentes et violentes de prurit, parfois de douleurs comme des piqûres d'épingle. Depuis trois semaines, il s'est formé partant de ce premier point et au-dessus de lui, une traînée inflammatoire dans laquelle on perçoit d'autres petites indurations; l'aspect de la peau à leur niveau est un peu rougeâtre. Pas d'hypertrophie des ganglions des régions correspondantes. Nous détachons la croutelle, et par la pression, nous obtenons un liquide hématique foncé. Son examen microscopique à l'état frais, et après coloration au Giemsa, ne nous permet pas d'aperçevoir de microbe, ni de parasite, mais nous notons une éosinophilie très marquée, environ un éosinophile sur trois globules blancs. Une numération globulaire du sang périphérique pris

Annales de Parasitologie, $\mathrm{T}, \mathrm{II}, \mathrm{N}^{\circ} 4 .-$ Octobre 1924, p. 322. 
au doigt ne nous donne pas par contre d'éosinophilie : polynucléaires : 80, grands mononucléaires : 11 , lymphocytes : 3 , éosinophilcs : 3 . L'affection étant peu étendue et bien limitée, l'éosinophilie locale nous faisant croire à un parasitisme local, nous conseillons au malade l'ablation de sa lésion qui est acceptée et pratiquée le soir même par le médecin-major Christiani. Toute la bande inflammatoire pouvant être pincée en bloc et soulevée des tissus profonds, la lésion est nettement comprise dans le tissu dermique et est détachée facilement. Guérison par Fremière intention. Nous sectionnons transversalement la pièce fraîche dans ses diverses parties; nous aperçevons alors sur une des coupes de la partie nodulaire principale une petite pellicule épaisse, molle et brunâtre de $2 \mathrm{~mm}$. de long, qui nous semble être une douve: l'examen direct au microscope confirme ce diagnostic. Le parasite est fixé au Bouin entre lames et coloré au carmin chlorhydrique. Des coupes histologiques en série faites sur la lésion, ne nous permettent pas de retrouver d'autre individu, mais nous précisent la localisation du parasite dans les parties moyennes du derme; la loge qu'il occupait est en partie libre, en partie occupée par de la fibrine, englobant des globules rouges et des polynucléaires surtout éosinophiles ainsi que quelques lymphocytes. Sur toute, l'étendue de la coupe, entre les faisceaux conjonctifs, entre les cellules graisseuses, parfois engainant les artérioles et les veinules et même des filets nerveux, on retrouve des cellules inflammatoires nombreuses; il n'y a nulle part d'abcès nettement formé.

Nous remercions M. le professeur agrégé Joyeux d'avoir bien voulu nous confirmer pour le parasite le diagnostic de Fasciola hepatica ; " il s'agit d un jeune distome à organes génitaux incomplètement développés ; la Iréparation comparée à une collection de jeunes Faciola hepatica montre la parfaite ressemblance de toutes ces formes $»$.

Ce parasitisme n'a rien qui puisse étonner étant donnée l'extrême fréquence de la $F$. hepatica dans les organes des animaux de boucherie au Maroc, où ils ne sont pas considérés pour cela, ainsi qu'en beaucoup d'autres pays d'ailleurs, comme impropres à la consommation. Nous rappellerons que la malade, qui a fait le sujet d'une communication de Dunet pour obstruction cholédocienne par grande douve du foie, avait fait un séjour prolongé à Fès, où elle avait d'ailleurs ressenti les premiers symptômes de son affection. En Afrique du Nord également, Maury et Pélissier ont observè un autre cas d'obstruction du cholédoque par douve à Alger.

Le mécanisme de l'infection est assez difficile à préciser ; il est admis que les animaux et accidentellement l'homme (Brumpt) se contaminent en avalant les kystes de cercairjes, que la jeune douve sort du kyste dans le tube digestif, traverse sa paroi, séjourne dans la cavité péritonéale d'où elle arrive aux "canaux 
biliaires qu'elle atieint en traversant la capsule du foie "Verdun supposait que la douve pénètre dans les vaisseaux à l'état de cercaire et a proposé des mécanismes divers pour expliquer les diverses localisations erratiques. Peut-être, dans certains cas, pourrait-il y avoir pénétration directe, et le mécanisme serait-il à rapprocher, au moins pour les localisations périphériques, de l'infestation par les bilharzies dont la cercaire est infectante et pénètre habituellement à travers la peau.

\section{Bibliographie}

Brumpt (E.). - Traité de parasitologie, Paris, 1922.

Chevaluier (J.). - Sur un cas de distomatose humaine. Thèse de Paris, 1924.

Dunet (Ch.). - Obstruction cholédocienne par grande douve du foie. C. R. Soc. Chir. de Lyon, 17 janvier 1924.

Gosset et Le Norr. - Douve vivante dans le cholédoque extraite par cholédocotomie. Guérison. Bull. et Mém. Soc. Nat. Chirurgie. L, nº 3, 2 février 1924.

Grall et Clarac. - Traité de pathologie exotique.

Maury (E.) et PÉlissier (R.). - Obstruction du cholédoque par douve du foie. Presse médicale, n 20, 8 mars 1924.

Taufflieb. - Distomatose à Fasciola hepatica chez l'homme et chez l'animal. Thèse de Lyon 1916 (1).

Laboraloire de l'Hôpital régional indigène Cocard.

(1) Cette thèse contient la bibliographie jusqu'en 1916. 